\title{
Foliated Schwarz symmetry for the nodal solution at the second minimax level
}

\author{
Kui Li ${ }^{1}$ - Zhitao Zhang ${ }^{2,3}$
}

Received: 16 July 2015 / Accepted: 28 April 2018 / Published online: 12 May 2018

(C) Fondazione Annali di Matematica Pura ed Applicata and Springer-Verlag GmbH Germany, part of Springer Nature 2018

\begin{abstract}
We study the second minimax level $\lambda_{2}$ of the eigenvalue problem for the scalar field equation in $\mathbb{R}^{N}$. By using the tool of polarization, we prove that every nodal solution at the second minimax level is foliated Schwarz symmetric. As a consequence, we prove an open problem of Perera and Tintarev (Annali di Matematica Pura ed Applicata (4) 194(1):131144, 2015).
\end{abstract}

Keywords Second minimax level $\cdot$ Nodal solution $\cdot$ Foliated Schwarz symmetry $\cdot$ Maximum principle

Mathematics Subject Classification $35 \mathrm{~J} 20 \cdot 35 \mathrm{~J} 61 \cdot 35 \mathrm{P} 30$

\section{Introduction}

In this paper, we consider variational problems of the form

$$
\inf _{\gamma \in \Gamma_{2}} \max _{u \in \gamma\left(S^{1}\right)} J(u)
$$

Supported by National Natural Science Foundation of China 11771428, 11688101, 11325107, 11331010.

Zhitao Zhang

zzt@math.ac.cn

Kui Li

likui@zzu.edu.cn

1 School of Mathematics and Statistics, Zhengzhou University, Zhengzhou 450001, Henan, People's Republic of China

2 HLM, CEMS, HCMS, Academy of Mathematics and Systems Science, Chinese Academy of Sciences, Beijing 100190, People's Republic of China

3 School of Mathematical Sciences, University of Chinese Academy of Sciences, Beijing 100049, People's Republic of China 
where $\Gamma_{2}:=\left\{\gamma \in C\left(S^{1}, \mathcal{M}\right): \gamma(-\theta)=-\gamma(\theta), \forall \theta \in S^{1}\right\}, S^{1}:=\left\{y \in \mathbb{R}^{2}:|y|=1\right\}, \mathcal{M}$ is a $C^{2}$ connected sub-manifold of a Hilbert space $H, \mathcal{M}=-\mathcal{M}$ and $J: H \rightarrow \mathbb{R}$ is a $C^{1}$ functional. For some differential equations, we want to show that any nodal solution which attains the above minimax level is foliated Schwarz symmetric with respect to some point $P \in S^{N-1}$.

Let $\Omega \subseteq \mathbb{R}^{N}$ be a symmetric domain, a continuous function $u(x): \Omega \rightarrow \mathbb{R}$ is called to be foliated Schwarz symmetric with respect to some point $P \in S^{N-1}=\left\{y \in \mathbb{R}^{N}:|y|=1\right\}$ if $u$ depends on $r=|x|>0$ and $\theta=\arccos \left(\frac{x}{|x|} \cdot P\right)$ and $u$ is nonincreasing in $\theta$ for any fixed $r>0$.

We focus on the topic of the eigenvalue problem for the scalar field equation

$$
-\Delta u+V(x) u=\lambda|u|^{p-2} u, \quad u \in H^{1}\left(\mathbb{R}^{N}\right),
$$

where $N \geq 3, p \in\left(2,2^{*}\right]\left(2^{*}=\frac{2 N}{N-2}\right), V \in L^{\infty}\left(\mathbb{R}^{N}\right)$.

For $u \in H^{1}\left(\mathbb{R}^{N}\right)$, let

$$
J(u)=\int_{\mathbb{R}^{N}}\left[|\nabla u|^{2}+V(x) u^{2}\right] \mathrm{d} x, \quad I(u)=\int_{\mathbb{R}^{N}}|u|^{p} \mathrm{~d} x .
$$

Thus, we define

$$
\mathcal{M}=\left\{u \in H^{1}\left(\mathbb{R}^{N}\right): I(u)=1\right\}, \quad \lambda_{2}:=\inf _{\gamma \in \Gamma_{2}} \max _{u \in \gamma\left(S^{1}\right)} J(u),
$$

where $\Gamma_{2}:=\left\{\gamma \in C\left(S^{1}, \mathcal{M}\right): \gamma(-\theta)=-\gamma(\theta), \forall \theta \in S^{1}\right\}$.

We use the tool of polarization (see $[2,3,8,9,14]$ ) to prove that any sign changing solution of (1.1) on $\mathcal{M}$ corresponding to $\lambda=\lambda_{2}$ is foliated Schwarz symmetric.

Theorem 1.1 Suppose that $p \in\left(2,2^{*}\right], \lambda_{2}>0$ and that $V \in L^{\infty}\left(\mathbb{R}^{N}\right)$ is radially symmetric. If $u$ is a nodal solution of (1.1) on $\mathcal{M}$ corresponding to $\lambda=\lambda_{2}$, then $u$ is foliated Schwarz symmetric with respect to some point $P \in S^{N-1}$, where $S^{N-1}=\left\{y \in \mathbb{R}^{N}:|y|=1\right\}$.

Remark 1.2 As $N=2,2^{*}=+\infty$, then for $2<p<+\infty$, Theorem 1.1 still holds.

Remark 1.3 Let $V \in L^{\infty}\left(\mathbb{R}^{N}\right), \lambda_{2}>0$ and $u$ be a nodal solution of (1.1) on $\mathcal{M}$ corresponding to $\lambda=\lambda_{2}$. Assume that $\Omega=\mathbb{R}^{N}$ and $\beta$ is the number defined for the equation (1.1) with $\lambda=1$ in [2]. Then, by scaling methods, we have

$$
\beta \leq\left(\frac{1}{2}-\frac{1}{p}\right) \lambda_{2}^{\frac{p}{p-2}} .
$$

Perera and Tintarev [9] proposed an open problem which states that if $V$ is radially symmetric, whether every solution of (1.1) at level $\lambda_{2}$ is foliated Schwarz symmetry or not. Here, we answer this open problem when the solution is nodal. In [10], they obtain the existence of nonradial nodal solution of (1.1) under some conditions on $V(x)$. In this paper, we can show that this solution is foliated Schwarz symmetric with respect to some point $P \in S^{N-1}$.

Corollary 1.4 Suppose that $p \in\left(2,2^{*}\right), V \in L^{\infty}\left(\mathbb{R}^{N}\right)$ is radially symmetric and satisfies

$$
V(x) \geq 0 \forall x \in \mathbb{R}^{N}, \quad \lim _{|x| \rightarrow \infty} V(x)=V^{\infty}>0 .
$$

Suppose that $W(x):=V^{\infty}-V(x) \in L^{\frac{p}{p-2}}\left(\mathbb{R}^{N}\right)$ satisfies

$$
W(x) \geq c_{0} e^{-a|x|}, \forall x \in \mathbb{R}^{N},|W|_{\frac{p}{p-2}}<\left(2^{\frac{p}{p-2}}-1\right) \lambda_{1}^{\infty},
$$


where $c_{0}$, a are some positive constants and

$$
\lambda_{1}^{\infty}:=\inf _{u \in \mathcal{M}} \int_{\mathbb{R}^{N}}\left[|\nabla u|^{2}+V^{\infty} u^{2}\right] \mathrm{d} x .
$$

Then, (1.1) has a nodal solution on $\mathcal{M}$ corresponding to $\lambda=\lambda_{2}$, which is foliated Schwarz symmetric with respect to some point $P \in S^{N-1}$.

For $u, v \in H^{1}\left(\mathbb{R}^{N}\right)$, we define the inner product as follows

$$
(u, v)=\int_{\mathbb{R}^{N}} \nabla u \nabla v \mathrm{dx}+\int_{\mathbb{R}^{N}} u v \mathrm{~d} x, \quad \forall u, v \in H^{1}\left(\mathbb{R}^{N}\right),
$$

and the corresponding norm is denoted by $\|u\|$. For $1 \leq p \leq+\infty$ and $f \in L^{p}\left(\mathbb{R}^{N}\right)$, we denote by $|f|_{p}$ the usual $L^{p}$-norm of $f, f^{+}=\max \{f, 0\}$ and $f^{-}=\max \{-f, 0\}$.

This paper is organized as follows: in Sect. 2, we give some preliminaries; in Sect. 3, we prove Theorem 1.1.

\section{Preliminaries}

In this section, we give some preliminaries. Let $\mathcal{H}$ be all the closed affine halfspaces in $\mathbb{R}^{N}$ and $\mathcal{H}_{0}=\{H: H \in \mathcal{H}, 0 \in \partial H\}$. For $H \in \mathcal{H}$, let $\sigma_{H}: \mathbb{R}^{N} \rightarrow \mathbb{R}^{N}$ be the reflection with respect to the boundary of $H$, and for a measurable function $u: \mathbb{R}^{N} \rightarrow \mathbb{R}$, let

$$
u_{H}(x)= \begin{cases}\max \left\{u(x), u\left(\sigma_{H}(x)\right)\right\}, & x \in H, \\ \min \left\{u(x), u\left(\sigma_{H}(x)\right)\right\}, & x \in \mathbb{R}^{N} \backslash H\end{cases}
$$

be the polarization of $u$ with respect to $H$ (see [2]).

Remark 2.1 From the definition of the polarization, it is easy to check that $u_{H}$ is measurable.

The following definition can be found in [4] or [12].

Definition 2.2 Let $\Omega \subseteq \mathbb{R}^{N}$ be a symmetric domain, a continuous function $u(x): \Omega \rightarrow \mathbb{R}$ is said to be foliated Schwarz symmetric with respect to some point $P \in S^{N-1}=\left\{y \in \mathbb{R}^{N}\right.$ : $|y|=1\}$ if $u$ depends on $r=|x|>0$ and $\theta=\arccos \left(\frac{x}{|x|} \cdot P\right)$ and $u$ is nonincreasing in $\theta$ for any fixed $r>0$.

Remark 2.3 Let $\mathcal{H}_{P}:=\left\{H: H \in \mathcal{H}_{0}\right.$ and $\left.P \in \stackrel{H}{H}\right\}$. The symmetrization $A^{P}$ of a set $A \subseteq S_{r}^{N-1}(0)=\partial B_{r}(0)$ with respect to $P$ is defined as the closed geodesic ball in $S_{r}^{N-1}(0)$ centered at $r P$ which satisfies $H^{N-1}\left(A^{P}\right)=H^{N-1}(A)$, where $H^{N-1}$ is the $N-1$-dimensional Hausdorff measure (see [1]). For a continuous function $u(x): \Omega \rightarrow \mathbb{R}$, the foliated Schwarz symmetrization $u_{P}: \Omega \rightarrow \mathbb{R}$ of u with respect to $P$ is defined by the condition

$$
\left\{u_{P} \geq t\right\} \cap S_{r}^{N-1}(0)=\left[\{u \geq t\} \cap S_{r}^{N-1}(0)\right]^{P}, \quad \forall r>0, t \in \mathbb{R} .
$$

One can check that $u_{P}$ is measurable and that $u$ is foliated Schwarz symmetric with respect to $P$ if and only if $u=u_{P}$ (See [14], p. 214).

The next two Lemmata can be found in [2], we omit their proofs. 
Lemma 2.4 For every measurable function $u: \mathbb{R}^{N} \rightarrow \mathbb{R}$ and for every $H \in \mathcal{H}$, there holds

$$
\left(u_{H}\right)^{+}=\left(u^{+}\right)_{H},\left(u_{H}\right)^{-}=\left(u^{-}\right)_{H} .
$$

In the following, we may simply write $u_{H}^{ \pm}$due to Lemma 2.4 .

Lemma 2.5 If $u \in W^{1, p}\left(\mathbb{R}^{N}\right)$ and $v \in L^{p}\left(\mathbb{R}^{N}\right)$ for some $1 \leq p<+\infty$, then for any $H \in \mathcal{H}$, we have

$$
u_{H} \in W^{1, p}\left(\mathbb{R}^{N}\right), \quad v_{H} \in L^{p}\left(\mathbb{R}^{N}\right),
$$

and

$$
\int_{\mathbb{R}^{N}}\left|\nabla u^{ \pm}\right|^{p} \mathrm{~d} x=\int_{\mathbb{R}^{N}}\left|\nabla u_{H}^{ \pm}\right|^{p} \mathrm{~d} x, \quad \int_{\mathbb{R}^{N}}\left|v^{ \pm}\right|^{p} \mathrm{~d} x=\int_{\mathbb{R}^{N}}\left|v_{H}^{ \pm}\right|^{p} \mathrm{~d} x .
$$

Moreover, if $U: \mathbb{R}^{N} \rightarrow \mathbb{R}$ is measurable and radially symmetric, and if

$$
\int_{\mathbb{R}^{N}}|U|\left|v^{ \pm}\right|^{p} \mathrm{~d} x<+\infty
$$

then for any $H \in \mathcal{H}_{0}$, we have

$$
\int_{\mathbb{R}^{N}} U\left|v^{ \pm}\right|^{p} \mathrm{~d} x=\int_{\mathbb{R}^{N}} U\left|v_{H}^{ \pm}\right|^{p} \mathrm{~d} x .
$$

Lemma 2.6 Suppose that $P \in S^{N-1}$ and $u \in C\left(\mathbb{R}^{N}\right)$. If $u_{H}(x)=u(x)$ for any $H \in \mathcal{H}_{P}$, then $u$ is foliated Schwarz symmetric with respect to $P$.

Proof According to Definition 2.2, we only need to prove that if $\left|x_{1}\right|=\left|x_{2}\right|$ and $\theta_{1}=$ $\arccos \left(\frac{x_{1}}{\left|x_{1}\right|} \cdot P\right) \leq \theta_{2}=\arccos \left(\frac{x_{2}}{\left|x_{2}\right|} \cdot P\right)$, then $u\left(x_{1}\right) \geq u\left(x_{2}\right)$.

If $\theta_{1}<\theta_{2}$, then $x_{1}$ is closer to $P$ than $x_{2}$; hence, there exists $H \in \mathcal{H}_{P}$ such that $x_{1} \in H$ and $\sigma_{H}\left(x_{1}\right)=x_{2}$. Since $u_{H}\left(x_{i}\right)=u\left(x_{i}\right)$ for $i=1,2$, we have $u\left(x_{1}\right) \geq u \circ \sigma_{H}\left(x_{1}\right)=u\left(x_{2}\right)$ by the definition of $u_{H}$.

If $\theta_{1}=\theta_{2}$, then there exists a sequence $\left\{\tilde{H}_{n}\right\}_{n=1}^{\infty} \subseteq \mathcal{H}_{P}$ with $x_{1} \in \tilde{H}_{n}$ and $\sigma_{\tilde{H}_{n}}\left(x_{1}\right) \rightarrow x_{2}$ as $n \rightarrow \infty$. Since $u\left(x_{1}\right) \geq u \circ \sigma_{\tilde{H}_{n}}\left(x_{1}\right)$, we have $u\left(x_{1}\right) \geq \lim _{n \rightarrow \infty} u \circ \sigma_{\tilde{H}_{n}}\left(x_{1}\right)=u\left(x_{2}\right)$.

\section{Proof of Theorem 1.1}

For $\lambda \in \mathbb{R}$, let $K_{\lambda}=\left\{u \in \mathcal{M}:\left.J\right|_{\mathcal{M}} ^{\prime}(u)=0, J(u)=\lambda\right\}$.

Lemma 3.1 If $\gamma_{0} \in \Gamma_{2}$ satisfying $\max _{\theta \in S^{1}} J\left(\gamma_{0}(\theta)\right)=\lambda_{2}$, then there exists $\theta_{0} \in S^{1}$ such that $\left.J\right|_{\mathcal{M}} ^{\prime}\left(\gamma_{0}\left(\theta_{0}\right)\right)=0$ and $J\left(\gamma_{0}\left(\theta_{0}\right)\right)=\lambda_{2}$.

Proof Let $\Sigma=\left\{\theta \in S^{1}: J\left(\gamma_{0}(\theta)\right)=\lambda_{2}\right\}$. Then, $\Sigma$ and $\gamma_{0}(\Sigma)$ are compact. If $\left.J\right|_{\mathcal{M}} ^{\prime}\left(\gamma_{0}(\theta)\right) \neq 0$ for any $\theta \in \Sigma$, then there exist $\varepsilon, \delta>0$ such that

$$
\forall u \in J^{-1}\left(\left[\lambda_{2}-2 \varepsilon, \lambda_{2}+2 \varepsilon\right]\right) \cap\left(\gamma_{0}(\Sigma)\right)_{3 \delta}:\left\|\left.J\right|_{\mathcal{M}} ^{\prime}(u)\right\| \geq \frac{8 \varepsilon}{\delta},
$$

where $\left(\gamma_{0}(\Sigma)\right)_{3 \delta}$ is the $3 \delta$ neighbor of $\gamma_{0}(\Sigma)$ in $\mathcal{M}$. Therefore, by Deformation Lemma (see [11] or [15]), there exists $\eta: C([0,1] \times \mathcal{M}, \mathcal{M})$ such that

(i) $\eta\left(1, J^{\lambda_{2}+\varepsilon}\right) \cap J\left(\left(\gamma_{0}(\Sigma)\right)_{\delta}\right) \subset J^{\lambda_{2}-\varepsilon}$,

(ii) $\eta(t,-u)=-\eta(t, u), \forall t \in[0,1], u \in \mathcal{M}$, 
(iii) $J(\eta(\cdot, u))$ is nonincreasing in $\mathbb{R}$ for any $u \in \mathcal{M}$.

Let $\tilde{\gamma_{0}}(\theta)=\eta\left(1, \gamma_{0}(\theta)\right)$, then $\tilde{\gamma_{0}} \in \Gamma_{2}$ by (ii). Since $\gamma_{0}\left(S^{1}\right) \subseteq J^{\lambda_{2}}$, we have $\sup J\left(\tilde{\gamma_{0}}\left(S^{1}\right)\right)<\lambda_{2}$ by (i) and (iii), this contradicts to the definition of $\lambda_{2}$. $\theta \in S^{1}$

For $u, v \in H^{1}\left(\mathbb{R}^{N}\right) \backslash\{0\}, v^{ \pm} \neq 0$, define

$$
\widehat{u}=\frac{u}{|u|_{p}} \text { and } \gamma_{v}(\cos (t), \sin (t))=\frac{\cos (t) \widehat{v^{+}}+\sin (t) \widehat{v^{-}}}{\left(|\cos (t)|^{p}+|\sin (t)|^{p}\right)^{\frac{1}{p}}},
$$

then

$$
\gamma_{v} \in \Gamma_{2}
$$

Now, we prove another Lemma which is similar to Proposition 4.2 of [2].

Lemma 3.2 Suppose that $\lambda_{2}>0$ and (1.1) has a nodal solution on $\mathcal{M}$ corresponding to $\lambda=\lambda_{2}$. Then,

$$
\lambda_{2}=\inf _{u \in \mathcal{M}, u^{ \pm} \neq 0} \max _{t \in[0,2 \pi]} \frac{J\left(\widehat{u^{+}}\right) \cos ^{2}(t)+J\left(\widehat{u^{-}}\right) \sin ^{2}(t)}{\left(|\cos (t)|^{p}+|\sin (t)|^{p}\right)^{\frac{2}{p}}} .
$$

Moreover, if $u \in \mathcal{M}$ satisfies $u^{ \pm} \neq 0$ and

$$
\max _{t \in[0,2 \pi]} \frac{J\left(\widehat{u^{+}}\right) \cos ^{2}(t)+J\left(\widehat{u^{-}}\right) \sin ^{2}(t)}{\left(|\cos (t)|^{p}+|\sin (t)|^{p}\right)^{\frac{2}{p}}}=\lambda_{2},
$$

then there exits $t_{0} \in[0,2 \pi]$ such that

$$
\left.J\right|_{\mathcal{M}} ^{\prime}\left(\gamma_{u}\left(\theta_{0}\right)\right)=0 \text { and } J\left(\gamma_{u}\left(\theta_{0}\right)\right)=\lambda_{2} .
$$

Proof Let

$$
\begin{aligned}
\lambda_{2}^{\prime} & =\inf _{u \in \mathcal{M}, u^{ \pm} \neq 0} \max _{t \in(0,2 \pi)} \frac{J\left(\widehat{u^{+}}\right) \cos ^{2}(t)+J\left(\widehat{u^{-}}\right) \sin ^{2}(t)}{\left(|\cos (t)|^{p}+|\sin (t)|^{p}\right)^{\frac{2}{p}}} \\
& =\inf _{u \in \mathcal{M}, u^{ \pm} \neq 0} \max _{S^{1}} J\left(\gamma_{u}\right) .
\end{aligned}
$$

Hence,

$$
\lambda_{2}^{\prime} \geq \lambda_{2}
$$

On the other hand, let $u_{0}$ be a nodal solution of (1.1) on $\mathcal{M}$ corresponding to $\lambda=\lambda_{2}$, and then

$$
-\Delta u_{0}+V(x) u_{0}=\lambda_{2}\left|u_{0}\right|^{p-2} u_{0} .
$$

Testing above equation with $u_{0}^{+}$and $u_{0}^{-}$and integrating over $\mathbb{R}^{N}$ give

$$
J\left(u_{0}^{ \pm}\right)=\lambda_{2}\left|u_{0}^{ \pm}\right|_{p}^{p}, \quad J\left(\widehat{u_{0}^{ \pm}}\right)=\frac{J\left(u_{0}^{ \pm}\right)}{\left|u_{0}^{ \pm}\right|_{p}^{2}}=\lambda_{2}\left|u_{0}^{ \pm}\right|_{p}^{p-2} .
$$

Therefore, according to the Hölder inequality and the positivity of $\lambda_{2}$, we have

$$
\begin{aligned}
\lambda_{2}^{\prime} & \leq J\left(\gamma_{u_{0}}(\cos (t), \sin (t))\right)=\frac{J\left(\widehat{u_{0}^{+}}\right) \cos ^{2}(t)+J\left(\widehat{u_{0}^{-}}\right) \sin ^{2}(t)}{\left(|\cos (t)|^{p}+|\sin (t)|^{p}\right)^{\frac{2}{p}}} \\
& =\lambda_{2} \frac{\left|u_{0}^{+}\right|_{p}^{p-2} \cos ^{2}(t)+\left|u_{0}^{-}\right|_{p}^{p-2} \sin ^{2}(t)}{\left(|\cos (t)|^{p}+|\sin (t)|^{p}\right)^{\frac{2}{p}}} \leq \lambda_{2}\left(\left|u_{0}^{+}\right|_{p}^{p}+\left|u_{0}^{-}\right|_{p}^{p}\right)^{\frac{p-2}{p}}=\lambda_{2} .
\end{aligned}
$$


Hence,

$$
\lambda_{2}=\lambda_{2}^{\prime}
$$

If

$$
\max _{t \in(0,2 \pi)} \frac{J\left(\widehat{u^{+}}\right) \cos ^{2}(t)+J\left(\widehat{u^{-}}\right) \sin ^{2}(t)}{\left(|\cos (t)|^{p}+|\sin (t)|^{p}\right)^{\frac{2}{p}}}=\max _{S^{1}} J\left(\gamma_{u}\right)=\lambda_{2},
$$

then Lemma 3.1 implies that there exits $t_{0} \in[0,2 \pi]$ such that

$$
\left.J\right|_{\mathcal{M}} ^{\prime}\left(\gamma_{u}\left(\theta_{0}\right)\right)=0 \text { and } J\left(\gamma_{u}\left(\theta_{0}\right)\right)=\lambda_{2}
$$

Lemma 3.3 If $\lambda_{2}>0, V \in L^{\infty}\left(\mathbb{R}^{N}\right)$ is symmetric and $u_{0} \in K_{\lambda_{2}}$ is nodal, then $u_{0 H}$ is also nodal and $u_{0 H}$ belongs to $K_{\lambda_{2}}$ for any $H \in \mathcal{H}_{0}$.

Proof In the following, we fixed a $H \in \mathcal{H}_{0}$. Since $u_{0}$ satisfies (1.1) with $\lambda=\lambda_{2}$ and $V \in L^{\infty}\left(\mathbb{R}^{N}\right)$, we have $u_{0} \in C^{1, \alpha}\left(\mathbb{R}^{N}\right)$ for any $\alpha \in(0,1)$ by elliptic regularity (see [6]). Hence,

$$
u_{0 H}=\frac{1}{2} u_{0}+\frac{1}{2} u_{0} \circ \sigma_{H}+\frac{1}{2} \chi_{H}\left|u_{0}-u_{0} \circ \sigma_{H}\right|-\frac{1}{2}\left(1-\chi_{H}\right)\left|u_{0}-u_{0} \circ \sigma_{H}\right| \in C\left(\mathbb{R}^{N}\right) .
$$

Since $u_{0}$ is nodal, we have that $u_{0 H}$ is nodal and $\left\{y \in \mathbb{R}^{N}:\left|u_{0 H}(y)\right|=0\right\} \neq \emptyset$.

Note that

$$
\begin{aligned}
& J\left(\gamma_{u_{0}}(\cos (t), \sin (t))\right)=\frac{J\left(\widehat{u_{0}^{+}}\right) \cos ^{2}(t)+J\left(\widehat{u_{0}^{-}}\right) \sin ^{2}(t)}{\left(|\cos (t)|^{p}+|\sin (t)|^{p}\right)^{\frac{2}{p}}}, \\
& J\left(\gamma_{u_{0 H}}(\cos (t), \sin (t))\right)=\frac{J\left(\widehat{u_{0 H}^{+}}\right) \cos ^{2}(t)+J\left(\widehat{u_{0 H}^{-}}\right) \sin ^{2}(t)}{\left(|\cos (t)|^{p}+|\sin (t)|^{p}\right)^{\frac{2}{p}}} .
\end{aligned}
$$

Lemmas 2.4 and 2.5 give

$$
J\left(\widehat{u_{0}^{+}}\right)=J\left(\widehat{u_{0 H}^{+}}\right), J\left(\widehat{u_{0}^{-}}\right)=J \widehat{\left(u_{0 H}^{-}\right)},
$$

hence

$$
\begin{gathered}
J\left(\gamma_{u_{0}}(\cos (t), \sin (t))\right)=J\left(\gamma_{u_{0 H}}(\cos (t), \sin (t))\right), \\
\max _{t \in[0,2 \pi]} J\left(\gamma_{u_{0}}(\cos (t), \sin (t))\right)=\max _{t \in[0,2 \pi]} J\left(\gamma_{u_{0 H}}(\cos (t), \sin (t))\right)=\lambda_{2} .
\end{gathered}
$$

On the other hand,

$$
\begin{aligned}
& J\left(\gamma_{u_{0 H}}(\cos (t), \sin (t))\right) \\
& =J\left(\gamma_{u_{0}}(\cos (t), \sin (t))\right) \\
& =\lambda_{2} \frac{\left|u_{0}^{+}\right|_{p}^{p-2} \cos ^{2}(t)+\left|u_{0}^{-}\right|_{p}^{p-2} \sin ^{2}(t)}{\left(|\cos (t)|^{p}+|\sin (t)|^{p}\right)^{\frac{2}{p}}} \\
& =\lambda_{2} \frac{\left|u_{0 H}^{+}\right|_{p}^{p-2} \cos ^{2}(t)+\left|u_{0 H}^{-}\right|_{p}^{p-2} \sin ^{2}(t)}{\left(|\cos (t)|^{p}+|\sin (t)|^{p}\right)^{\frac{2}{p}}} \\
& \leq \lambda_{2}
\end{aligned}
$$


with equality holds if and only if $|\tan (t)|=\frac{\left|u_{0}^{+}\right|_{p}}{\left|u_{0}^{-}\right|_{p}}=\frac{\left|u_{0 H}^{+}\right|_{p}}{\left|u_{0 H}^{-}\right|_{p}}$. Thus, Lemma 3.2 implies that there is a $t_{0} \in[0,2 \pi]$ such that

$$
\left|\tan \left(t_{0}\right)\right|=\frac{\left|u_{0}^{+}\right|_{p}}{\left|u_{0}^{-}\right|_{p}}=\frac{\left|u_{0 H}^{+}\right|_{p}}{\left|u_{0 H}^{-}\right|_{p}} \text { and } \frac{\cos \left(t_{0}\right) \widehat{u_{0 H}^{+}}+\sin \left(t_{0}\right) \widehat{u_{0 H}^{-}}}{\left(\left|\cos \left(t_{0}\right)\right|^{p}+\left|\sin \left(t_{0}\right)\right|^{p}\right)^{\frac{1}{p}}} \in K_{\lambda_{2}},
$$

hence

$$
u_{0 H}=u_{0 H}^{+}-u_{0 H}^{-} \text {or }\left|u_{0 H}\right|=u_{0 H}^{+}+u_{0 H}^{-} \in K_{\lambda_{2}} .
$$

If $\left|u_{0 H}\right| \in K_{\lambda_{2}}$, then

$$
-\Delta\left|u_{0 H}\right|+V(x)\left|u_{0 H}\right|=\lambda_{2}\left|u_{0 H}\right|^{p-1} \geq 0 .
$$

Since $V \in L^{\infty}\left(\mathbb{R}^{N}\right)$, elliptic regularities ([6]) give $\left|u_{0 H}\right| \in C^{1, \alpha}\left(\mathbb{R}^{N}\right)$ for any $\alpha \in(0,1)$. Maximal principle (see [5] or [7]) and the fact that $\left\{y \in \mathbb{R}^{N}:\left|u_{0 H}(y)\right|=0\right\} \neq \emptyset$ imply that $\left|u_{0 H}\right| \equiv 0$, and this contradicts with the fact that $\left|u_{0 H}\right|_{p}=|u|_{p}=1$.

Therefore,

$$
u_{0 H} \in K_{\lambda_{2}} .
$$

Now, we turn to the proof of Theorem 1.1.

Proof If $u$ is nodal and solves (1.1) with $\lambda=\lambda_{2}$, then $u_{H}$ also solves (1.1) with $\lambda=\lambda_{2}$ for any $H \in \mathcal{H}_{0}$ by Lemma 3.3. Since $V \in L^{\infty}\left(\mathbb{R}^{N}\right)$, elliptic regularities (see [6]) give $u, u_{H} \in$ $C^{1, \alpha}\left(\mathbb{R}^{N}\right)$ for any $\alpha \in(0,1)$. Choose $P \in S^{N-1}$ such that $u(P)=\max \left\{u(x): x \in S^{N-1}\right\}$ and we will show that $u$ is foliated Schwarz symmetric with respect to $P$.

Fix any $H \in \mathcal{H}_{P}$. In $\stackrel{\circ}{H}:=H \backslash \partial H$, we have

$$
\left|u-u \circ \sigma_{H}\right|=2 u_{H}-\left(u+u \circ \sigma_{H}\right),
$$

therefore,

$$
\begin{aligned}
- & \Delta\left|u-u \circ \sigma_{H}\right|+V(x)\left|u-u \circ \sigma_{H}\right| \\
& \left.=2\left(-\Delta u_{H}+V(x) u_{H}\right)-\left(-\Delta u+V(x) u-\Delta u \circ \sigma_{H}+V(x) u \circ \sigma_{H}\right)\right) \\
& =2 \lambda_{2}\left|u_{H}\right|^{p-2} u_{H}-\left(\lambda_{2}|u|^{p-2} u+\lambda_{2}\left|u \circ \sigma_{H}\right|^{p-2} u \circ \sigma_{H}\right) \\
& \left.=\lambda_{2}\left[\left(\left|u_{H}\right|^{p-2} u_{H}-|u|^{p-2} u\right)+\left(\left|u_{H}\right|^{p-2} u_{H}-\left|u \circ \sigma_{H}\right|^{p-2} u \circ \sigma_{H}\right)\right)\right] \geq 0,
\end{aligned}
$$

hence

$$
\left|u-u \circ \sigma_{H}\right|>0 \text { in } \stackrel{\circ}{H} \text { or }\left|u-u \circ \sigma_{H}\right|=0 \text { in } \stackrel{\circ}{H} .
$$

Since $P \in \stackrel{\circ}{H}$, we have

$$
u(P) \geq u \circ \sigma_{H}(P),
$$

hence

$$
u>u \circ \sigma_{H} \text { in } \stackrel{\circ}{H} \text { or } u=u \circ \sigma_{H} \text { in } \stackrel{\circ}{H} \text {. }
$$

Therefore, 


$$
u_{H}=u \text {. }
$$

Thus, for any $H \in \mathcal{H}_{P}$, we have

$$
u_{H}=u \text {. }
$$

Lemma 2.6 implies $u$ is foliated Schwarz symmetric with respect to $P$, and hence we get Theorem 1.1.

Remark 3.4 The condition on $V(x)$ in Theorem 1.1 can be relaxed to " $V \in L_{l o c}^{\infty}$ ". Replacing $H^{1}\left(\mathbb{R}^{N}\right)$ by

$$
H=\left\{u \in H^{1}\left(\mathbb{R}^{N}\right): \int_{\mathbb{R}^{N}}\left[|\nabla u|^{2}+(1+|V(x)|) u^{2}\right] \mathrm{d} x<\infty\right\},
$$

the inner product on $H$ can be defined as follows

$$
(u, v)=\int_{\mathbb{R}^{N}} \nabla u \nabla v \mathrm{~d} x+\int_{\mathbb{R}^{N}}(1+|V(x)|) u v \mathrm{~d} x, \quad \forall u, v \in H .
$$

It is clear that $H$ is a Hilbert space (see [13]).

Then, we define

$$
\mathcal{M}=\{u \in H: I(u)=1\}, \quad \lambda_{2}:=\inf _{\gamma \in \Gamma_{2}} \max _{u \in \gamma\left(S^{1}\right)} J(u),
$$

where $\Gamma_{2}:=\left\{\gamma \in C\left(S^{1}, \mathcal{M}\right): \gamma(-\theta)=-\gamma(\theta), \forall \theta \in S^{1}\right\}$.

Using the similar methods, we can prove Theorem 1.1 under the assumption " $V \in L_{l o c}^{\infty}$ ".

Acknowledgements The authors would like to thank the referees very much for their careful reading and useful comments on the previous version of this paper.

\section{References}

1. Albert Baernstein, I.I., Taylor, B.A.: Spherical rearrangements, subharmonic functions, and $*$-functions in $n$-space. Duke Math. J. 43(2), 217-439 (1976)

2. Bartsch, T., Weth, T., Willem, M.: Partial symmetry of least energy nodal solutions to some variational problems. J. d'Anal. Mathmat. 96(1), 1-18 (2005)

3. Bartsch, T., Wang, Z.Q., Zhang, Z.T.: On the Fučik point spectrum for Schrödinger operators on $\mathbb{R}^{N}$. J. Fixed Point Theory Appl. 5(2), 305-317 (2009)

4. Damascelli, L., Pacella, F.: Symmetry results for cooperative elliptic systems via linearization. SIAM J. Math. Anal. 45(3), 1003-1026 (2013)

5. Evans, L.C.: Partial Differential Equations, Graduate Students in Mathematics, vol. 19. AMS, Providence (1998)

6. Gilbarg, D., Trudinger, N.S.: Elliptic Partial Differential Equations of Second Order, 1998th edn. Springer, Berlin (2001). (Reprint of the 1998 ed.)

7. Han, Q., Lin, F.: Equations, Elliptic Partial Differential. Lecture Notes, Courant Institute of Mathematical Sciences, New York (1997)

8. Pacella, F.: Symmetry results for solutions of semilinear elliptic equations with convex nonlinearities. J. Funct. Anal. 192(1), 271-282 (2002)

9. Perera, K., Tintarev, C.: On the second minimax level of the scalar field equation and symmetry breaking. Annali di Matematica Pura ed Applicata (4) 194(1), 131-144 (2015)

10. Perera, K., Tintarev, C.: A nodal solution of the scalar field equation at the second minimax level. Bull. Lond. Math. Soc. 46(6), 1218-1225 (2014)

11. Struwe, M.: Variational Methods. Springer, Berlin (1990)

12. Weth, T.: Symmetry of solutions to variational problems for nonlinear elliptic equations via reflection methods. Jahresbericht der Deutschen Mathematiker-Vereinigung 112(3), 119-158 (2010)

13. Willem, M.: Minimax Theorems. Springer, Berlin (1996) 
14. Zhang, Z.: Variational, Topological, and Partial Order Methods with Their Applications. Developments in Mathematics, vol. 29. Springer, Heidelberg (2013)

15. Zhong, C.K., Fan, X.L.: Introduction to Nonlinear Functional Analysis. Lanzhou University Press, Lanzhou (2004). (in Chinese) 